\title{
Thermodynamic evaluation and modeling of proton and water exchange associated with benzamidine and berenil binding to ß-trypsin
}

M.T. Pereira ${ }^{1,2}$,

J.M. Silva-Alves²,

A. Martins-José ${ }^{3}$,

J.C.D. Lopes $^{3}$ and M.M. Santoro ${ }^{2}$
${ }^{1}$ Centro de Desenvolvimento da Tecnologia Nuclear, 2Departamento de Bioquímica e Imunologia, Instituto de Ciências Biológicas, ${ }^{3}$ NEQUIM, Departamento de Química, Instituto de Ciências Exatas, Universidade Federal de Minas Gerais, Belo Horizonte, MG, Brasil
Correspondence

M.M. Santoro

Departamento de Bioquímica e

Imunologia, UFMG

Av. Antonio Carlos, 6627

31270-901 Belo Horizonte, MC

Brasil

E-mail: santoro@icb.ufmg.br

Received September 22, 2004 Accepted July 28, 2005

\section{Abstract}

Serine-proteases are involved in vital processes in virtually all species. They are important targets for researchers studying the relationships between protein structure and activity, for the rational design of new pharmaceuticals. Trypsin was used as a model to assess a possible differential contribution of hydration water to the binding of two synthetic inhibitors. Thermodynamic parameters for the association of bovine B-trypsin (homogeneous material, observed 23,294.4 \pm 0.2 $\mathrm{Da}$, theoretical $23,292.5 \mathrm{Da}$ ) with the inhibitors benzamidine and berenil at $\mathrm{pH} 8.0,25^{\circ} \mathrm{C}$ and with $25 \mathrm{mM} \mathrm{CaCl}_{2}$, were determined using isothermal titration calorimetry and the osmotic stress method. The association constant for berenil was about 12 times higher compared to the one for benzamidine (binding constants are $\mathrm{K}=596,599 \pm$ 25,057 and 49,513 $\pm 2,732 \mathrm{M}^{-1}$, respectively; the number of binding sites is the same for both ligands, $\mathrm{N}=0.99 \pm 0.05$ ). Apparently the driving force responsible for this large difference of affinity is not due to hydrophobic interactions because the variation in heat capacity $(\Delta \mathrm{Cp})$, a characteristic signature of these interactions, was similar in both systems tested $\left(-464.7 \pm 23.9\right.$ and $-477.1 \pm 86.8 \mathrm{~J} \mathrm{~K}^{-1} \mathrm{~mol}^{-1}$ for berenil and benzamidine, respectively). The results also indicated that the enzyme has a net gain of about 21 water molecules regardless of the inhibitor tested. It was shown that the difference in affinity could be due to a larger number of interactions between berenil and the enzyme based on computational modeling. The data support the view that pharmaceuticals derived from benzamidine that enable hydrogen bond formation outside the catalytic binding pocket of ß-trypsin may result in more effective inhibitors.

\section{Introduction}

The objectives of the study of the interactions of proteins with ligands are to increase our understanding of their function, their importance in physiological processes, the
Key words

- Benzamidine

- Berenil

- Calorimetry

- Protein modeling

- Trypsin

- Osmotic stress 
mation about the relationship between protein structure and activity has been obtained in X-ray crystallography studies on many proteins and with modern bioinformatic techniques (1). Complex molecular organizations can now be studied at the atomic level. The notion that proteins craft well-defined binding sites that are custom-tailored to their ligand remains central to analyses of biological recognition and drug discovery (2). Nevertheless, the contact between a protein and its coupling with any other co-solute cannot be explained based on random steric interactions. Different forms of electrostatic forces necessarily act in intermolecular or interatomic interactions (3), reflecting on the catalytic efficacy of an enzyme or the specificity of any protein.

ß-Trypsin was chosen for the present study due to its importance as a protease model for the serine-protease class. Proteins of this class play an important role in physiological and pathological processes (4). Furthermore, trypsin is reasonably stable and relatively easy to obtain and purify. It was considered to be an exclusively digestive enzyme expressed in the pancreas (5), where it is produced as the zymogen trypsinogen. Four trypsinogen isoforms are known so far: trypsinogen 1 or cationic trypsinogen, trypsinogen 2 or anionic trypsinogen, trypsinogen 3 or mesotrypsinogen (6), and trypsinogen 4, identified in brain cells (7). Several diseases are associated with deregulation of the cationic form, especially pancreatitis (8). Recent studies have shown that trypsin is widely expressed at low levels in various non-pancreatic epithelial tissues, vascular endothelial cells, leukocytes, and neurons, and is overexpressed in some kinds of cancer cells and tissues $(9,10)$.

Cationic pancreatic trypsinogen originates the B-trypsin isoform after a hydrolysis that liberates the first six residues of the peptide chain. Additional cleavages produce the isoforms $\psi, \alpha, \gamma, \delta$, and others with decreasing amidase activity (11). This au- tolytic process can regulate the activity of the enzyme which is also exerted through natural inhibitors such as bovine pancreatic trypsin inhibitor and several others described in the literature. After zymogen cleavage, the active $\beta$-trypsin form acquires higher plasticity, maintaining the spatial distribution of the several segments practically intact (12). Differential scanning calorimetry characterization at acid $\mathrm{pH}$ plus the $\mathrm{pH}$ titration of native and unfolded forms allowed the estimation of the stability of this enzyme at acid and neutral $\mathrm{pH}$ (13).

Data obtained by computational techniques, spectroscopy or established laboratory methods have provided more information on the interactions between solutes and solvent. The involvement of water molecules, helping to define conformational states of the protein through hydrogen bonds, is very important because intra- and intermolecular interactions can modulate the elasticity and plasticity of proteins, which are fundamental for their action (14-16). The involvement of hydration water molecules, by inclusion or exclusion, has been considered to be a driving force in the interactions involving low charge complementarities (17). Binding of water to hemoglobin, for example, is the determinant step in the mechanism of allosteric regulation (18). An analytical method known as osmotic stress has been developed based on this inclusion/exclusion process for situations of low macromolecular concentrations (19-21), being extensively applied to analyze the hydration water evolved in the interaction of macromolecules $(19,22)$.

Isothermal titration microcalorimetry and the osmotic stress method with thermodynamic parameters were used in the present study to see how benzamidine and berenil (Figure 1), two structurally distinct but chemically similar low-molecular weight synthetic inhibitors, interact with bovine cationic Btrypsin. Benzamidine is known to be a competitive inhibitor of trypsin. It is also considered to be a model compound for the basic 
amino acids that represent the primary specificity of this enzyme (23). The results obtained for berenil are compared to those for benzamidine. The involvement of water molecules and protons associated with the binding of these inhibitors is also described.

\section{Material and Methods}

\section{Purification of ß-trypsin}

A commercial bovine trypsin $\left(\operatorname{Sigma}^{\circledR}\right.$, St. Louis, MO, USA) was submitted to chromatography $(730 \mathrm{ml}$ on a $50-\mathrm{cm}$ high column, $4 \mathrm{~cm}$ in inner diameter) with SE-Sephadex-C50 and eluted with $50 \mathrm{mM}$ sodium formiate. The $\mathrm{pH}$ was adjusted to 3.50 with formic acid. The enzyme was eluted with a linear increasing $\mathrm{NaCl}$ gradient ( 0.1 to 0.3 M), containing $25 \mathrm{mM} \mathrm{CaCl}_{2}$ and $1 \mathrm{mM}$ benzamidine. The active fractions containing B-trypsin were identified (24) and combined. The pool was lyophilized, resuspended in $1 \mathrm{mM} \mathrm{HCl}$, dialyzed for $4 \mathrm{~h}$ against the same solution, and for another $2 \mathrm{~h}$ in milliQ water at $4^{\circ} \mathrm{C}$ in both cases. All low-molecular weight components were thus eliminated from the pooled effluent. The pooled effluent was lyophilized once more and 2.5- to 3.0-mg aliquots of the enzyme were stored at $-10^{\circ} \mathrm{C}$ until use. When necessary, the fraction was dissolved in the desired buffer in an ice bath immediately before each experiment, in volumes reaching final concentrations of about 1.0 to $3.0 \mathrm{mg} / \mathrm{ml}$. Concentrations were determined by absorbance measurements at $280 \mathrm{~nm}$ with the molar extinction coefficient of $37,000 \mathrm{M}^{-1} \mathrm{~cm}^{-1}(25)$. Titration of active sites were performed with $\mathrm{p}$ nitrophenyl-p-guanidinobenzoate- $\mathrm{HCl}$ (26). One sample was then submitted to mass spectrometry to confirm ß-trypsin homogeneity.

\section{Microcalorimetry}

Titration of enzyme-inhibitor interactions was carried out with an isothermal titration calorimeter (VP-ITC, MicroCal LLC, Northampton, MA, USA) (usable cell volume of $1.42 \mathrm{ml}$ ), using Pipes, HEPES, tricine and Tris buffers $\left(50 \mathrm{mM}\right.$, with $25 \mathrm{mM} \mathrm{CaCl}_{2}, \mathrm{pH}$ 8.0). Benzamidine or berenil concentrations were determined during the experiment by the injection and data acquisition procedure according to the equipment manual. Briefly: [L] $>30$ [Enz]; an initial injection of $1 \mu \mathrm{l}$ was discarded, and 20 to 30 injections of 3-5 $\mu \mathrm{l}$ resulted in the complete saturation of the enzyme binding site. The data were analyzed by fitting a single-site binding isotherm (27). After a single experiment at controlled temperature, the following parameters were determined: the reaction equilibrium constant and consequently the variation of Gibbs energy $\left(\Delta \mathrm{G}_{\text {bind }}\right)$, the stoichiometric relationship between the reagent species, or the number of binding sites concerning the association between proteins and ligands, and the enthalpy variation of the association $\left(\Delta \mathrm{H}_{\text {bind }}\right)$. The entropy variation of the association $\left(\Delta \mathrm{S}_{\text {bind }}\right)$ was then calculated.

\section{Osmotic stress}

Osmotic stress experiments were carried out with $\beta$-trypsin and the inhibitors benzamidine and berenil $(50 \mathrm{mM}$ Tris, with 25 $\mathrm{mM} \mathrm{CaCl} 2, \mathrm{pH} 8.0$, at $25^{\circ} \mathrm{C}$ ). Two sets of isothermal titration calorimetry experiments were performed for each inhibitor with concentrations of glucose or glycine ranging

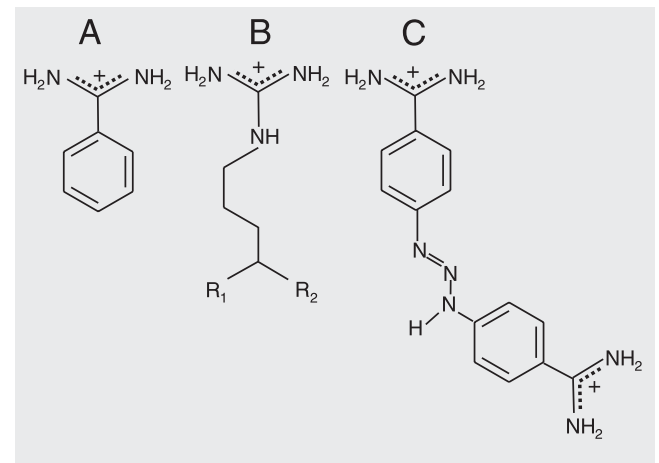

Figure 1. Structures of benzamidine $(A)$, side chain of arginine (B) and berenil (C). 
from 0.25 to 1.00 osm, here used as compatible osmolites.

\section{Docking and minimization}

The complexes between ß-trypsin and the ligands berenil, $\mathrm{p}$-aminobenzamidine and benzamidine were formed by replacing the benzamidine contained in the crystallographic structure deposited in the Protein Data Bank (<http://www.rscb.org > PDB ID 1CE5), using the superimposal procedure of the INSIGHTII program (MSI). The ligand charges were calculated by the MNDO method from the MOPAC package (28). The complexes thus constructed were studied with the AutoDock program, version 3.0 (29), using a method based on the Genetic Algorithm to search for the best position for the ligands at the active site of $\beta$-trypsin. One hundred genetic algorithm runs were used, with a population of 50 individuals, a gene mutation rate of 0.02 and crossover rates of 0.8 . The affinity grid was centered at Ser ${ }^{195}$ with dimensions of 48 x 48 x $48 \AA$ and a grid spacing of $0.5 \AA$, including the catalytic site and its surroundings (16). The most stable conformation according to the docking energy was transferred to the SYBYL program (30) in which optimization of the geometry of the complexes and of the isolated ligands and trypsin was performed. The 4.0 value was used for the distancedependent dielectric constant, with a cutoff of $14.0 \AA$.

\section{Results and Discussion}

The participation of water molecules not as a solvent but as an adjuvant in protein action has deserved special attention in the last few years (31). The degree of hydration seems to define states of higher or lower structural flexibility for the protein (17). It was proposed that about 300 water molecules are associated with trypsin (32). A recent study from our laboratory (33) dem- onstrated, on the basis of the variation of the Stokes radius, the occurrence of an intermediate state between native and denatured forms, a molten globule. It was estimated that 770 associated water molecules occur if the variation in the volume of the protein is due only to included/excluded water molecules.

Titrations of the enzyme with the ligands benzamidine and berenil were performed on purified ß-trypsin. A typical isotherm of these titrations is shown in Figure 2. $\Delta \mathrm{G}, \Delta \mathrm{H}, \Delta \mathrm{S}$ and the stoichiometric relationships for the experiments in Tris buffer are shown in Table 1. These results refer to $0.0 \mathrm{osm}$ (without any addition of co-solute or osmolite). The results for $\Delta \mathrm{G}$ in the titration with benzamidine confirm literature data regarding this parameter $(23,25,34)$. It is noteworthy that the value of the association constant for berenil was 12 times higher than the constant for benzamidine.

Some energetic contribution (absorbed or released heat) specific for the ionization/ association process may contribute to the effects of the enzyme-ligand association itself. Such a contribution, or dependence of the observed result, can be a consequence of association/dissociation processes of ionizable groups in the enzyme, exchanged with the buffer. The variation in the protonation state of the residues involved in the association process can be obtained from the number of protons exchanged between the enzyme and the buffer by the linked Wyman equation $(19,35): \Delta \mathrm{H}_{\mathrm{obs}}=\Delta \mathrm{H}_{\text {bind }}+\mathrm{nH}^{+} \mathrm{x}$ $\Delta \mathrm{H}_{\text {dis }}$, where $\Delta \mathrm{H}_{\mathrm{obs}}$ is the value measured in the experiment, $\Delta \mathrm{H}_{\text {bind }}$ is the enthalpy value of the enzyme-inhibitor association itself, $\mathrm{nH}^{+}$corresponds to the number of protons exchanged, and $\Delta \mathrm{H}_{\mathrm{dis}}$ corresponds to the values of the variation in the enthalpy of the ionization of the buffers. $\Delta \mathrm{H}_{\text {bind }}$ and $\mathrm{nH}^{+}$can be calculated from the intercept and the slope of the curve in the graphic representation of $\Delta \mathrm{H}_{\text {obs }}$ versus $\Delta \mathrm{H}_{\text {dis. }}$. The titrations were performed in four different buffers covering a 
wide range of enthalpy of the proton dissociation $\left(\Delta \mathrm{H}_{\mathrm{dis}}\right)$ interval: Pipes $\left(\Delta \mathrm{H}_{\mathrm{dis}}=11.45\right.$ $\mathrm{kJ} / \mathrm{mol})$; HEPES $\left(\Delta \mathrm{H}_{\mathrm{dis}}=21.01 \mathrm{~kJ} / \mathrm{mol}\right)$; tricine $\left(\Delta \mathrm{H}_{\mathrm{dis}}=31.96 \mathrm{~kJ} / \mathrm{mol}\right)$, and Tris $\left(\Delta \mathrm{H}_{\mathrm{dis}}\right.$ $=47.53 \mathrm{~kJ} / \mathrm{mol}$ ) (36). For the experiments (graph not shown) with benzamidine, we found that $\Delta \mathrm{H}_{\text {bind }}=-16.78 \pm 0.80$ and $\mathrm{nH}^{+}=$ $0.037 \pm 0.026 \mathrm{~kJ} / \mathrm{mol}$, and for the experiments with berenil $\Delta \mathrm{H}_{\text {bind }}=-18.20 \pm 3.78$ and $\mathrm{nH}^{+}=0.007 \pm 0.12 \mathrm{~kJ} / \mathrm{mol}$. These results indicate that there is no association or dissociation of acidic groups during binding of the two ligands.

The results shown in Table 1 (lines corresponding to $0.00 \mathrm{osm}$ ) indicate that there is a favorable enthalpic and entropic contribution to the formation of the $\beta$-trypsin-inhibitor complex. The enthalpic contribution can be analyzed on the basis of the equation $\delta \Delta \mathrm{H}$ $=\Delta \mathrm{Cp} \times \delta \mathrm{T}$ which, after integration, becomes: $\Delta \mathrm{H}=\Delta \mathrm{Cp} \times \mathrm{T}+$ one constant, for $\Delta \mathrm{Cp}$ constant in the temperature interval studied. The calculated values for the change in the heat capacity for the B-trypsin-inhibitor associations in Tris buffer are (graph not shown): $\Delta \mathrm{Cp}=-477.1 \pm 86.8 \mathrm{~J} \mathrm{~K}^{-1} \mathrm{~mol}^{-1}$ for benzamidine, and $\Delta \mathrm{Cp}=-464.7 \pm 23.9 \mathrm{~J} \mathrm{~K}^{-1}$ $\mathrm{mol}^{-1}$ for berenil. It should be observed that the value of $\Delta \mathrm{S}$ obtained in the absence of the osmolite (Table 1 ) is about 10 to 20 times lower than these $\Delta \mathrm{Cp}$ values.

As a consequence of the relationships of $\Delta \mathrm{H}, \Delta \mathrm{S}$ and $\mathrm{T}$ with $\Delta \mathrm{G}$, and of $\Delta \mathrm{H}$ with $\mathrm{T}$, $\delta \Delta \mathrm{H} / \delta \mathrm{T}=\Delta \mathrm{Cp}$ and $\delta(\mathrm{TAS}) / \delta \mathrm{T}=\Delta \mathrm{Cp}+\Delta \mathrm{S}$. When $\Delta \mathrm{S}$ becomes negligible with respect to $\Delta \mathrm{Cp}$ the dependence of $\Delta \mathrm{H}$ and $\mathrm{T} \Delta \mathrm{S}$ becomes a constant factor equal to $\Delta \mathrm{Cp}$, and $\Delta \mathrm{G}$ becomes relatively independent of $\mathrm{T}$. This is shown both by the benzamidine and berenil associations with ß-trypsin, as can be observed in Figure 3. The temperatures at which the $\Delta \mathrm{H}$ and $\Delta \mathrm{S}$ parameters become zero, $\mathrm{T}_{\mathrm{H}}$ and $\mathrm{T}_{\mathrm{S}}$ respectively, were calculated from the graphs: $\mathrm{T}_{\mathrm{H}}=-3.6^{\circ} \mathrm{C}$ and $\mathrm{T}_{\mathrm{S}}=$ $62.3^{\circ} \mathrm{C}$ for benzamidine, and $\mathrm{T}_{\mathrm{H}}=-11.9^{\circ} \mathrm{C}$ and $\mathrm{T}_{\mathrm{S}}=58.4^{\circ} \mathrm{C}$ for berenil.

The well-known effect of water mol-

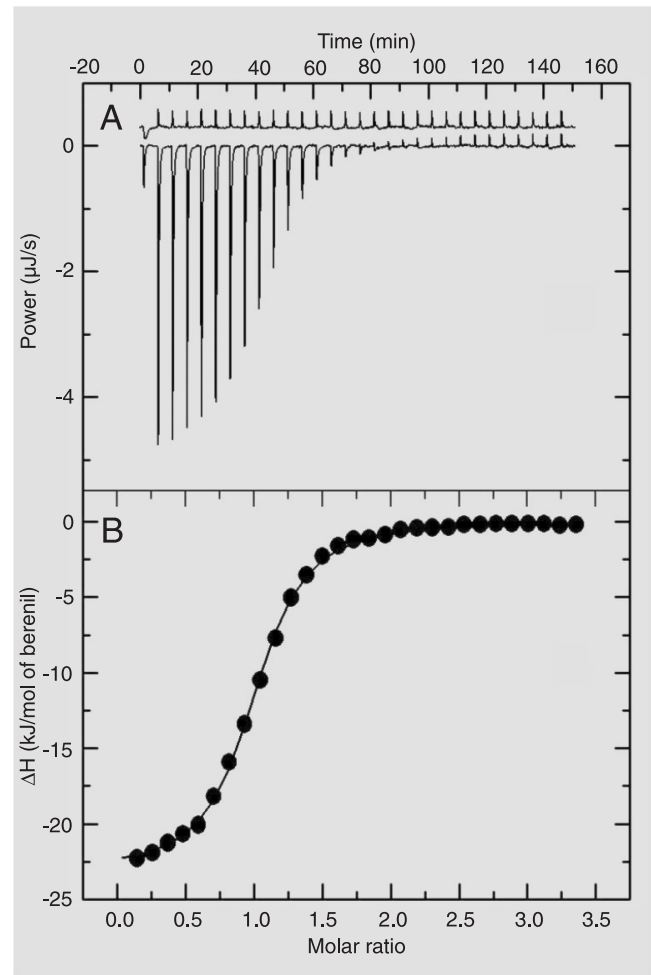

Figure 2. Isothermal titration calorimetry of B-trypsin with berenil. The data in this figure were obtained in $50 \mathrm{mM}$ Tris buffer, with $25 \mathrm{mM} \mathrm{CaCl}_{2}$, at $\mathrm{pH}$ $8.0,25^{\circ} \mathrm{C}$. $A$, The lower trace shows the raw data for the titration and the upper trace shows the heat of dilution of the berenil. $B$, Net isotherm of the titration obtained by subtracting the gross value of heat at each titration point by the linear regression of the dilution heat data of berenil.

Table 1. Energetic parameters of binding of the inhibitors benzamidine and berenil to B-trypsin.

\begin{tabular}{cccccc}
\hline & $\begin{array}{c}\text { Osmolality } \\
(\mathrm{osm})\end{array}$ & $\mathrm{N}$ & $\begin{array}{c}\Delta \mathrm{G} \\
(\mathrm{kJ} / \mathrm{mol})\end{array}$ & $\begin{array}{c}\Delta \mathrm{H} \\
(\mathrm{kJ} / \mathrm{mol})\end{array}$ & $\begin{array}{c}\Delta \mathrm{S} \\
\left(\mathrm{J} \mathrm{mol} \mathrm{I}^{-1}\right)\end{array}$ \\
\hline $\begin{array}{c}\text { Benzamidine as ligand } \\
\text { Glycine as osmolite }\end{array}$ & 0.00 & 0.957 & -26.79 & -15.17 & 38.99 \\
& 0.25 & 1.033 & -26.84 & -12.53 & 48.02 \\
& 0.50 & 1.028 & -26.13 & -7.08 & 63.93 \\
Glucose as osmolite & 1.00 & 0.954 & -25.70 & -6.48 & 64.50 \\
& 0.00 & 0.957 & -26.79 & -15.17 & 38.99 \\
Berenil as ligand & 0.38 & 1.126 & -26.74 & -15.15 & 38.89 \\
Glycine as osmolite & 0.75 & 0.986 & -26.17 & -11.44 & 49.43 \\
& & & & & \\
Glucose as osmolite & 0.00 & 0.990 & -32.95 & -17.16 & 52.98 \\
& 0.38 & 0.993 & -32.81 & -12.33 & 68.72 \\
& 0.75 & 0.994 & -32.17 & -12.31 & 66.64 \\
& 1.00 & 0.909 & -32.18 & -10.03 & 74.36 \\
& 0.00 & 0.990 & -32.95 & -17.16 & 52.98 \\
& 0.25 & 0.961 & -32.17 & -18.33 & 46.44 \\
& 0.50 & 0.945 & -31.96 & -14.10 & 59.93 \\
& 1.00 & 0.991 & -32.23 & -10.50 & 72.92
\end{tabular}

Although the osmolytes glycine and glucose have opposite effects on the dielectric constant of water, they have similar effects on the thermodynamic parameters of the binding process of the competitive inhibitors to B-trypsin. $\mathrm{N}=$ number of sites in the enzyme; $\Delta \mathrm{G}=$ variation of Gibbs energy; $\Delta \mathrm{H}=$ enthalpy variation; $\Delta \mathrm{S}=$ entropy variation. 
ecules on the structure $(15,16)$ and function of proteins, influencing the macromolecular dynamics, allows additional insight into the energetic aspects involved in the enzymeinhibitor interaction. This was done using the osmotic stress method (19-21). With the Gibbs-Duhem equation it can be demonstrated that, for a protein at infinite dilution (21) $\mathrm{d} \Delta \mathrm{G}=-\Delta \mathrm{N}_{\mathrm{ew}} \mathrm{d} \mu_{\mathrm{w}}$. In this equation $\Delta \mathrm{N}_{\mathrm{ew}}$ is the variation in the number of water molecules in the final macromolecular state minus the number in the initial state, ew is the deficit or excess of water molecules, and $\mu_{w}$ is the chemical potential of the water or another equivalent parameter. The equation can be written as dlnK/dlna ${ }_{\mathrm{w}}=\Delta \mathrm{N}_{\mathrm{ew}}=\mathrm{N}_{\mathrm{w}}^{\mathrm{EI}}-$ $\left(\mathrm{N}_{\mathrm{w}}^{\mathrm{E}}+\mathrm{N}_{\mathrm{w}}^{\mathrm{I}}\right)$. In this equation $\mathrm{N}_{\mathrm{w}}^{\mathrm{EI}}$ is the number of water molecules associated with the complex formed and $\mathrm{N}_{\mathrm{w}}^{\mathrm{E}}$ and $\mathrm{N}_{\mathrm{w}}^{\mathrm{I}}$ are the number of water molecules associated with the enzyme and with the inhibitor in the bath, respectively. The results obtained from the osmotic stress analyses are shown in Table
Figure 3. Dependence of $\Delta G$ and $\Delta \mathrm{H}$ on $\mathrm{T} \Delta \mathrm{S}$. Symbols: Circles for the ß-trypsin-benzamidine systems and triangles for the B-trypsin-berenil systems. The filled symbols indicate $\Delta \mathrm{H}$ values and the open symbols indicate $\Delta \mathrm{G}$ values. $\Delta \mathrm{G}=$ variation of Gibbs energy; $\Delta \mathrm{H}=$ enthalpy variation; $\mathrm{T} \Delta \mathrm{S}=$ temperature times entropy variation.

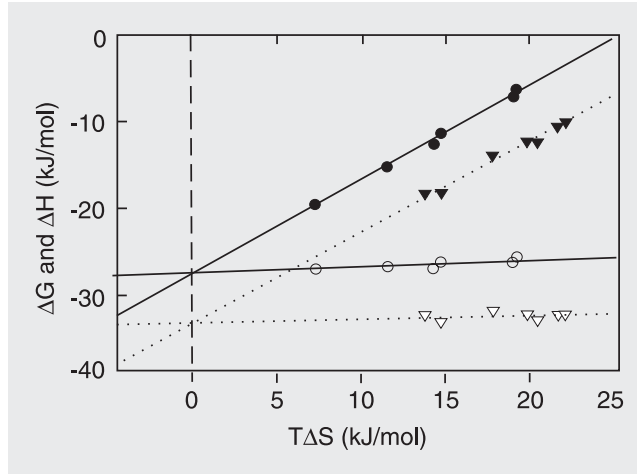

Figure 4. Dependence of $\ln (\mathrm{K} /$ $\mathrm{K}_{0}$ ) on the formation of the complex of B-trypsin with the inhibitors benzamidine and berenil as a function of the water activity (In $a_{w}$ ). Data referring to each inhibitor-osmolite set were normalized. The value of the slope is $21 \pm 3.4$ water molecules and the correlation coefficient is $r^{2}=$ 0.881 . Filled circles indicate data for benzamidine, and open circles indicate data for berenil.
1. The utilization of glucose and glycine as osmolites follows the strategy of Colombo and Bonilla-Rodriguez (37), in order to exclude a possible interference due to a change in the dielectric constant of the solution, since the two osmolites have opposite effects on this property. The data for each inhibitor as a function of the chemical potential of water were plotted and resulted in the same slopes within the experimental error. Consequently they resulted in an equal number of water molecules, regardless of the osmolite and the inhibitor tested. Because of this similarity and for a better statistical alignment of the results the values for the equilibrium constants were normalized and plotted; only one regression was calculated, as shown in Figure 4. From the slope of this curve the number of water molecules taken up from the bulk water during binding was estimated at $21.1 \pm 3.4$. Surfaces topologically distant from the catalytic site (16) are considered to be active participants in this inclusion. According to Parsegian et al. (20), "this result is characteristic of reactions with changes in the number of waters that are sequestered in sterically inaccessible pockets or cavities". Enzymes are known to be more stable when substrates and competitive inhibitors are present, and a tightening of the enzyme is linked to the formation of the corresponding adducts. In fact, the result observed, inclusion, involves the participation of distant surfaces in the process of inhibitor binding to the catalytic pocket per se since the exclusion of at least five water molecules would be expected to occur. According to computational modeling (38), the volume occupied by the side chain of arginine corresponds to the volume of six water molecules, while the corresponding volume for the lysine side chain is equivalent to five water molecules; in the latter case one water molecule stays in the catalytic pocket to compensate for a smaller side chain (16).

Theoretical studies of the binding of these two inhibitors to the enzyme were carried 
out hoping to use modeling data to explain the difference in the affinities of benzamidine and berenil for ß-trypsin. Furthermore, p-aminobenzamidine was included in the analysis because some experimental data are available in the literature $(25,34)$, and because its structure is related to those of the two ligands under study. Using the AutoDock and the SYBYL programs, the complexes formed between $\beta$-trypsin (R) and the mentioned inhibitors (L) were optimized (RL); the energies of the enzyme and the ligands isolated in the pharmacophoric conformation were evaluated ( $\mathrm{Ri}$ and $\mathrm{Li}$, respectively) as also were the energies after optimization of the geometries ( $\mathrm{Rf}$ and Lf, respectively). These results are shown in Table 2, which also shows the values for energies of perturbation (Ep), interaction (Ei), and binding $(\mathrm{Eb})$, calculated according to the following equations: $\mathrm{Ep}=\mathrm{EpR}+\mathrm{EpL}=(\mathrm{Rf}-\mathrm{Ri})+(\mathrm{Lf}$ - $\mathrm{Li}) ; \mathrm{Ei}=\mathrm{RL}-\mathrm{Ri}-\mathrm{Li}$ and $\mathrm{Eb}=\mathrm{Ei}+\mathrm{Ep}$.

The calculated energies (Ep, Ei and Eb) for benzamidine and p-aminobenzamidine are reasonably coincident, which is the result of the structural similarity of the two compounds. Among the calculated binding energies, only berenil had a negative value. This is due to the kind of perturbations caused by the other two ligands at the binding site of trypsin (EpR), according to the force field applied. The calculated enegies reflect only the intermolecular interaction between the ligand and enzyme, after optimization with the SYBYL package. It is also worth noting that berenil exhibits the highest interaction energy. The fact that the benzamidine interaction energy was slightly more favorable than that of p-aminobenzamidine can be explained on the dispersion of the positive charge of the amidine group of the latter. This occurs because the amino group, an excellent electron donor, decreases the positive charge of the amidine group, thus reducing the interaction of this group with the carboxylate of $\mathrm{Asp}^{177}$ present at the bottom of the pocket of the S1 site.
However, calorimetric determinations contradict this result since the measured affinity of p-aminobenzamidine is about 4 times higher than that of benzamidine $(25,34)$. This discrepancy may be due to the methodology used in the calculations that does not take into account the effect of differential solvation of the complexes or of the isolated ligands and receptor. The variation in the heat capacity, $\Delta \mathrm{Cp}$, for both inhibitors suggests that the contribution of the hydrophobic effect should be negative for both compounds (39). $\Delta \mathrm{Cp}$ for benzamidine binding is $-469.4 \pm 51.4 \mathrm{~J} \mathrm{~mol}^{-1} \mathrm{~K}^{-1}$ (this work) and $-519 \mathrm{~J} \mathrm{~mol}^{-1} \mathrm{~K}^{-1}$ for p-aminobenzamidine (25). The solvation and desolvation effects of the ligands, receptor and the complexes, i.e., the changes in free energies to transfer the ligands from the bulky solution to the binding site, are exergonic. The fact that the positive charge of the amidine is more dispersed in the p-aminobenzamidine group should decrease the interaction force between this inhibitor and the water molecules. Consequently, the energetic cost related to the release of the water molecules around the ligand molecule, necessary to the binding, will be smaller for p-aminobenzamidine compared to benzamidine. Thus, p-aminobenzamidine is expected to present a more favorable binding constant than benzamidine, although the specific interaction inside the binding site is weakened by charge dispersion.

Table 2. Theoretical energetic components involved in the binding of B-trypsin to inhibitors $(\mathrm{kJ} / \mathrm{mol})$.

\begin{tabular}{lccccccccc}
\hline & $\mathrm{RL}$ & $\mathrm{Ri}$ & $\mathrm{Rf}$ & $\mathrm{Li}$ & $\mathrm{Lf}$ & $\mathrm{Ei}$ & $\mathrm{Eb}$ & $\mathrm{EpR}$ & $\mathrm{EpL}$ \\
\hline Benz & -2380.0 & -2348.7 & -2494.1 & 15.5 & 13.2 & -46.9 & 59.0 & 103.3 & 2.3 \\
pAmB & -2379.7 & -2348.4 & -2494.2 & 14.5 & 12.7 & -45.8 & 60.0 & 103.9 & 1.8 \\
Berenil & -2435.9 & -2366.1 & -2503.1 & 36.9 & 28.6 & -106.7 & -3.7 & 94.9 & 8.2
\end{tabular}

Benz $=$ benzamidine and $\mathrm{pAmB}=\mathrm{p}$-aminobenzamidine. $\mathrm{RL}=$ complex enzymeinhibitor after optimization of the structure; $\mathrm{Ri}$ and $\mathrm{Li}=$ the enzyme and the inhibitor isolated in the pharmacophoric conformation, respectively; Rf (for the enzyme) and Lf (for the ligands) $=$ the energies after optimization of the geometries; $\mathrm{Ep}, \mathrm{Ei}$, and $\mathrm{Eb}=$ the energies of perturbation, interaction and binding, respectively. 
The effects of charge dispersion on free energy of binding are two-fold and opposite: at the same time they weaken the interaction between the amidine group and the aspartic residue at the bottom of the pocket, that hinders the binding interaction, but they also weaken the interaction between the amidine group and solvent molecules, which favors the binding interaction. The free energy of binding from isothermal titration calorimetry studies indicates that the second effect should be more pronounced. However, these effects need to be investigated by computing solvent accessible surface areas and calculating free solvation energy in order to gain

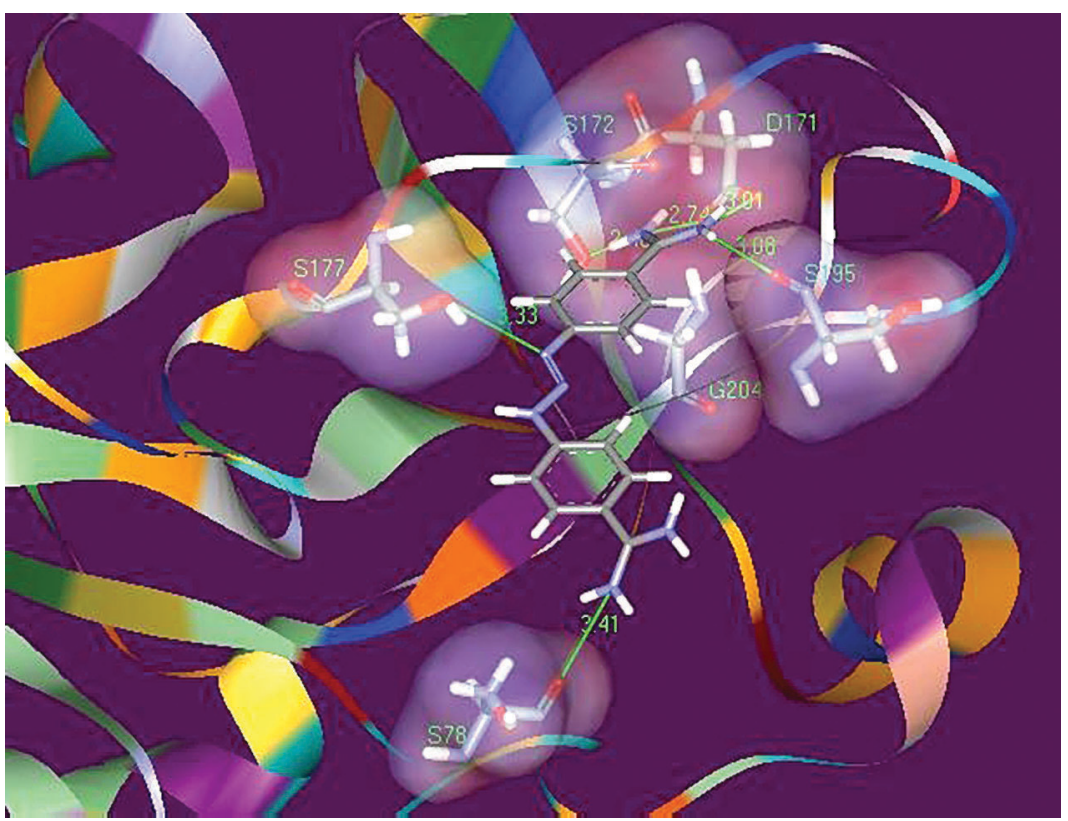

Figure 5. Interactions of berenil with residues located at or near the S1 catalytic site of Btrypsin. Only hydrogen bonds are shown. Berenil forms hydrogen bonds with Asp ${ }^{171}$, Ser ${ }^{195}$, Gly ${ }^{204}$, Ser ${ }^{177}$, and Ser ${ }^{78}$ residues. The residues Asp ${ }^{171}$, Ser ${ }^{195}$ and Gly ${ }^{204}$ are located at the bottom of $\mathrm{S} 1$ pocket. The Ser ${ }^{177}$ residue is located at the entrance and the $\mathrm{Ser}^{78}$ residue is located outside the catalytic site. some insight into the solvation and desolvation effects.

The participation of hydrogen bonds is another important factor in the stabilization of inhibitor-enzyme complexes. The present study evaluates the possibilities of this type of interaction among the molecules analyzed. In the simulations we observed that $\mathrm{p}$-aminobenzamidine can form an additional hydrogen bond with the $\mathrm{OH}$ group of the $\mathrm{Ser}^{177}$ residue. Figure 5 shows the arrangement of berenil in the pocket of the catalytic S1 site of B-trypsin after optimization and minimization of energies with the SYBYL program. Berenil shows several interactions with trypsin, some in the interior of the pocket with residues $\mathrm{Asp}^{171}$ and $\mathrm{Ser}^{195}$, and also with the $\mathrm{Ser}^{177}$, $\mathrm{His}^{40}$ and $\mathrm{Ser}^{78}$ residues located outside the catalytic site. Berenil shows a much more negative interaction energy than the two benzamidines and, despite presenting several contact points with the receptor, it shows a less positive perturbation energy. These results explain quantitatively the calorimetric data obtained and, most importantly, they show that benzamidine and berenil are promising molecules as starting points for the synthesis of chemical derivatives that can act as improved ligands or inhibitors for trypsin and other related serine proteases. Aditionally, many physiologically important serine proteases are in fact "trypsin-like" enzymes and the information developed here can have direct applications.

\section{Acknowledgments}

We thank one anonymous referee for useful suggestions and comments.

\section{References}

1. Czapinska H \& Otlewski J (1999). Structural and energetic determinants of the $\mathrm{S}_{1}$-site specificity in serine proteases. European Journal of Biochemistry, 260: 571-595.

2. Mu T, Lester HA \& Dougherty DA (2003). Different binding orientations for the same agonist at homologous receptors: a lock and key or a simple wedge? Journal of the American Chemical Association, 125: 6850-6851.

3. Naray-Szabo G \& Nagy P (1986). Electrostatic lock-and-key model for the study of biological isosterism: role of structural water in the binding of basic pancreatic trypsin inhibitor to beta-trypsin. Enzyme, 
36: 44-53.

4. Netzel-Arnett S, Hooper JD, Szabo R et al. (2003). Membrane anchored serine-proteases: a rapidly expanding group of cell surface proteolytic enzymes with potential roles in cancer. Cancer and Metastasis Reviews, 22: 237-258.

5. Rothman S, Liebow C \& Isenman L (2002). Conservation of digestive enzymes. Physiological Reviews, 82: 1-18.

6. Rinderknecht H, Renner IG, Abramson SB et al. (1984). Mesotrypsin: a new inhibitor-resistant protease from a zymogen in human pancreatic tissue and fluid. Gastroenterology, 86: 681-692.

7. Wiegand U, Corbach S, Minn A et al. (1993). Cloning of the cDNA encoding human brain trypsinogen and characterization of its product. Gene, 136: 167-175.

8. Kukor Z, Tóth M \& Tóth-Sahin M (2003). Human anionic trypsinogen: Properties of autocatalytic activation and degradation and implications in pancreatic diseases. European Journal of Biochemistry, 270: 2047-2058.

9. Koshikawa N, Hasegawa S, Nagashima $Y$ et al. (1998). Expression of trypsin by epithelial cells of various tissues, leukocytes, and neurons in human and mouse. American Journal of Pathology, 153: 937-944.

10. Stenman UH (2002). Tumor-associated trypsin inhibitor. Clinical Chemistry, 48: 1206-1209.

11. Foucault G, Seydoux F \& Yon J (1974). Comparative kinetic properties of $\alpha, \beta$ and $\psi$ forms of trypsin. European Journal of Biochemistry, 295: 295-302.

12. Kossiakoff AA, Chambers JL, Kay LM et al. (1977). Structure of bovine trypsinogen at $1.9 \AA$ resolution. Biochemistry, 16: 654-664.

13. Bittar ER, Caldeira FR, Santos AM et al. (2003). Characterization of B-trypsin at acid $\mathrm{pH}$ by differential scanning calorimetry. Brazilian Journal of Medical and Biological Research, 36: 1621-1627.

14. Santoro MM, Liu Y, Khan SMA et al. (1992). Increased thermal stability of proteins in the presence of natural occurring osmolytes. Biochemistry, 31: 278-283.

15. Fischer S \& Verma CS (1999). Binding of buried structural water increases the flexibility of proteins. Proceedings of the National Academy of Sciences, USA, 96: 9613-9615.

16. Helland R, Otlewski J, Sundheim O et al. (1999). The crystal structure of the complexes between bovine B-trypsin and ten $\mathrm{P}_{1}$ variants of BPTI. Journal of Molecular Biology, 287: 923-942.

17. Camacho CJ, Kimura KR, DeLisi C et al. (2000). Kinetics of desolvation-mediated protein-protein binding. Biophysical Journal, 78: 10941105.

18. Salvay AG, Grigera JR \& Colombo MF (2003). The role of hydration on the mechanism of allosteric regulation: in situ measurements of the oxygen-linked kinetics of water binding to hemoglobin. Biophysical Journal, 84: 564-570.

19. Colombo MF, Rau DC \& Parsegian VA (1992). Protein solvation in allosteric regulation: a water effect on hemoglobin. Science, 256: 655-659.

20. Parsegian VA, Rand RP \& Rau DC (2000). Osmotic stress, crowding, preferential hydration, and binding: A comparison of perspectives. Proceedings of the National Academy of Sciences, USA, 97: 3987-3992.

21. LiCata VJ \& Allewell NM (1998). Measuring hydration changes of proteins in solution: applications of osmotic stress and structurebased calculations. Methods in Enzymology, 295: 42-62.

22. Rand RP (2004). Probing the role of water in protein conformation and function. Philosophical Transactions of the Royal Society of London, Series B. Biological Sciences, 359: 1277-1284.

23. Mares-Guia M \& Shaw E (1965). Studies on the active center of trypsin. The binding of amidines and guanidines as models of the substrate side chain. Journal of Biological Chemistry, 240: 15791585.

24. Erlanger BF, Kokowsky N \& Cohen W (1961). The preparation and properties of two new chromogenic substrates of trypsin. Archives of Biochemistry and Biophysics, 95: 271-278.

25. Talhout R \& Engberts JBFN (2001). Thermodynamic analysis of binding of p-substituted benzamidines to trypsin. European Journal of Biochemistry, 268: 1554-1560.

26. Chase Jr T \& Shaw E (1967). p-Nitrophenyl-p-guanidinobenzoate$\mathrm{HCl}$ : a new active site titrant for trypsin. Biochemical and Biophysical Research Communications, 29: 508-514.

27. Wiseman T, Williston S, Brandt JF et al. (1989). Rapid measurement of binding constants and heats of binding using a new calorimeter. Analytical Biochemistry, 179: 131-135.

28. Stewart JJ (1990). MOPAC: A semi empirical molecular orbital program. Journal of Computer-Aided Molecular Design, 4: 1-105.

29. Goodsell DS, Morris GM \& Olson AJ (1996). Automated docking of flexible ligands: applications of AutoDock. Journal of Molecular Recognition, 9: 1-5.

30. Sybyl 6.5 (1999). Tripos Associates, St. Louis, MO, USA.

31. Papoian GA, Ulander J, Eastwood MP et al. (2004). From the cover: water in protein structure prediction. Proceedings of the National Academy of Sciences, USA, 101: 3352-3357.

32. Suurkuusk J (1974). Specific heat measurements on lysozyme, chymotrypsinogen, and ovalbumin in aqueous solution and in solid state. Acta Chemica Scandinavica B, 28: 409-417.

33. Martins NF, Ferreira E, Torres KC et al. (2003). The denaturation of alpha, beta and psi bovine trypsin at $\mathrm{pH}$ 3.0: evidence of intermediates. Protein and Peptide Letters, 10: 73-81.

34. Oliveira MGA, Rogana E, Rosa JC et al. (1993). Tyrosine 151 is part of the substrate activation binding site of bovine trypsin. Journal of Biological Chemistry, 268: 26893-26903.

35. Wyman Jr J (1964). Linked functions and reciprocal effects in hemoglobin: a second look. Advances in Protein Chemistry, 19: 223-286.

36. Fukada H \& Takahashi K (1998). Enthalpy and heat capacity changes for the proton dissociation of various buffer components in 0.1 M potassium chloride. Proteins, 33: 159-166.

37. Colombo MF \& Bonilla-Rodriguez GO (1996). The water effect on allosteric regulation of hemoglobin probed in water/glucose and water/glycine solutions. Journal of Biological Chemistry, 271: 48954899.

38. Gerstein M, Tsai J \& Levitt M (1995). The volume of atoms on the protein surface: Calculated from simulation, using Voronoi polyhedra. Journal of Molecular Biology, 249: 955-966.

39. Spolar RS, Ha JH \& Record Jr MT (1989). Role of the hydrophobic effect in stability of site-specific protein-DNA complexes. Proceedings of the National Academy of Sciences, USA, 86: 8382-8385. 